\title{
Numerical and experimental investigation of multilayer SS410 thin wall built by laser direct metal deposition
}

\author{
Yongjie Zhang, Gang Yu*, Xiuli He, Weijian Ning, Caiyun Zheng \\ Key Laboratory of Mechanics in Advanced Manufacturing, Institute of Mechanics, Chinese Academy of Sciences, Beijing 100190, People's Republic of China
}

\section{A R T I C L E I N F O}

\section{Article history:}

Received 18 January 2011

Received in revised form 5 July 2011

Accepted 8 August 2011

Available online 16 August 2011

\section{Keywords:}

Laser direct metal deposition

Finite element modeling

Stainless steel 410

\begin{abstract}
A B S T R A C T
The influence of thermal history on the microstructures and properties of a multilayer stainless steel 410 (SS410) thin wall built by laser direct metal deposition (LDMD) process was investigated experimentally and numerically. Thermal history at two specified points in the substrate was measured by thermocouples during the process. A three-dimensional (3D) finite element model was developed to study the thermal history of the deposited material for the laser direct metal deposition of multilayer thin wall. The simulated and measured thermal history indicated that the absorption and loss of heat tended to be close to equilibrium when the deposited material reached a certain height during the LDMD process. Different microstructure regions were formed due to the different thermal history the material experienced. The hardness distribution along the height centerline of the thin wall was measured. The results indicated that thermal history had an important effect on the microstructure, and consequently on the final properties.
\end{abstract}

(C) 2011 Published by Elsevier B.V.

\section{Introduction}

Laser direct metal deposition (LDMD), also known as the laser engineered net shaping (LENS) or laser rapid forming (LRF), is a promising direct manufacturing process. Stainless steel 410 (SS410) is an important class of martensitic stainless steel which is widely used in engineering such as seamless pipes, turbines, surgical tools and mixers of chemical products. Complex threedimensional (3D) SS410 parts having industrial applications can be manufactured directly from computer aided design (CAD) models by the LDMD process.

Some investigations on the thermal behavior, final microstructure and properties of laser direct metal deposited SS410 have been carried out by modeling and experiments. Wang et al. (2008) developed a 3D finite element model to analyze the temperature evolution in LENS deposition of ten layers SS410. They optimized the process parameters to maintain a steady melt pool size. Yin et al. (2008) studied the thermal behavior during LENS deposition of ten layers SS410 using a 2D transient finite element model. They evaluated the 2D model by comparing the results obtained from the 2D model and a previously developed 3D model. Due to the lack of available experimental data for SS410, both Wang et al. (2008) and Yin et al. (2008) validated their models with the experimental data for SS316. Wang et al. (2009) investigated

\footnotetext{
* Corresponding author. Tel.: +86 010 82544250; fax: +86 01082544250

E-mail address: gyu@imech.ac.cn (G. Yu).
}

the temperature distribution, cooling rate and melt pool size for different process parameters by model and experiments. Wang and Felicelli (2007) developed a 3D finite element model to study the effect of laser power and travel speed on the phase transformation and the consequent hardness of the laser deposition of ten layers SS410. They assumed that three possible phases could be present in the final part, including retained austenite, fresh martensite and tempered martensite. The results showed that the hardness in the upper region of the thin wall was higher than that in the lower region. Pratt et al. (2008) investigated the residual stresses in LENS SS410 thin walls by neutron diffraction method and finite element models. Krishna and Bandyopadhyay (2009) carried out surface modification of SS410 by laser surface-melting using LENS to study the influence of laser surface-melting parameters on microstructures. The results showed that the surface hardness increased due to the reduction of the retained austenite. It can be seen that most of these studies focus on the thin wall with no more than ten deposited layers. Additionally, few experimental investigations were carried out on the influence of thermal history on the microstructure and hardness of the laser deposited SS410 thin wall.

In this paper, a SS410 thin wall with 200 layers was built by LDMD process. Thermal history by thermocouples at two specified points in the substrate was obtained. A 3D finite element model was developed to simulate the temperature evolution during the process. The influence of thermal history on the final microstructure and microhardness distribution of the thin wall was also studied. 


\section{Mathematical modeling}

In this section, a mathematical model was used to simulate the transient temperature field during the deposition of multilayer SS410 thin wall.

\subsection{Assumptions}

To simplify the calculations, some assumptions are made for the model. They are summarized as follows:

A1. The laser energy distribution is a circular Gaussian as follows:

$q(r)=\frac{2 Q}{\pi \cdot r_{b}^{2}} \exp \left(-\frac{2 r^{2}}{r_{b}^{2}}\right)$

Where $Q$ is the laser power, $r_{b}$ is the laser beam radius.

For simplicity, the thermal load of the model is given in the form of the average thermal flux density that is:

$a q_{m}=\frac{a}{\pi \cdot r_{b}^{2}} \int_{0}^{r_{b}} q(r) \cdot 2 \pi r \cdot d r=\frac{0.865 a Q}{\pi \cdot r_{b}^{2}}$

Where $a$ is the absorptivity, $q_{m}$ represents the average thermal flux density, $q(r)$ is given by Eq. (1).

A2. A combined heat transfer coefficient $h_{c}$ is used to simulate the effect of radiation and convection. It can be calculated from the relation as follows (Alimardani et al., 2007):

$h_{c}=2.41 \times 10^{-3} \varepsilon T^{1.61}$

Where $\varepsilon$ is the emissivity, $T$ is temperature.

A3. Thermophysical properties of the materials are temperature-dependent and piecewise linear with the temperature.

\subsection{Governing equation}

The transient temperature distribution $T(x, y, z, t)$ can be calculated from heat transfer equation with appropriate boundary conditions. The governing equation based on heat transfer equation is expressed as:

$\frac{\partial(\rho c T)}{\partial t}=\frac{\partial}{\partial x}\left(k \frac{\partial T}{\partial x}\right)+\frac{\partial}{\partial y}\left(k \frac{\partial T}{\partial y}\right)+\frac{\partial}{\partial z}\left(k \frac{\partial T}{\partial z}\right)$

Where $\rho, c, t$ and $k$ are density, specific heat capacity, time and thermal conductivity, respectively.

\subsection{Initial and boundary conditions}

The initial temperature of the substrate and powder particles is room temperature. So the initial condition is

$T(x, y, z, 0)=T_{a}$

Where $T_{a}$ is the room temperature.

The convection and radiation boundary condition can be considered together as

$k \frac{\partial T}{\partial n}=h_{c}\left(T-T_{a}\right)$

Where $\vec{n}$ is the normal vector of the surface.

The effect of the moving laser beam can be considered as a surface heat source as follows:

$k\left(\frac{\partial T}{\partial n}\right)=a q_{m}$

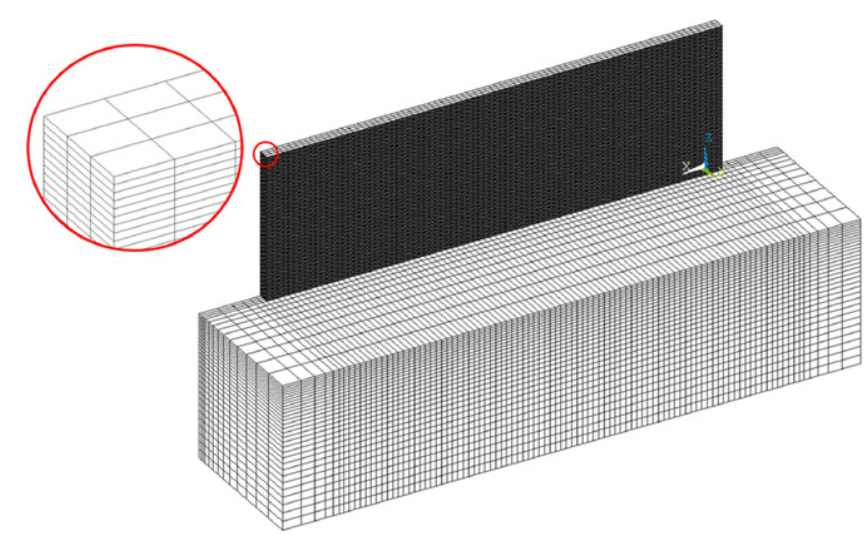

Fig. 1. Finite element mesh and geometry for the thermal calculations.

Table 1

Chemical composition of SS410 powder (wt\%).

\begin{tabular}{lllll}
\hline $\mathrm{C}$ & $\mathrm{Cr}$ & $\mathrm{Si}$ & $\mathrm{Mn}$ & $\mathrm{Fe}$ \\
\hline 0.10 & 12.0 & 1.52 & 0.15 & Bal. \\
\hline
\end{tabular}

\subsection{Numerical method}

A 3D finite element model based on above mathematical model was developed with the ANSYS parametric design language (APDL). Since the geometry and boundary conditions are symmetric, half of geometry can be used as computational domain to save calculation time. The geometry and finite element mesh used in the model are shown in Fig. 1. The model was meshed with eight-node brick elements SOLID70. A denser mesh was used in the laser heat areas.

The initial node temperature of substrate was room temperature. All the elements of thin wall were inactive and not contributing to the solution. The continuous additions of metal powders were considered as successive discrete activation of new set of elements. The boundary conditions were updated dynamically with the activation of new elements. When the deposition process was finished, the part cooled down to room temperature.

\section{Experimental procedures}

The powder used in the experiment was water atomized SS410 powder with a particle size range of 30-90 $\mu \mathrm{m}$. Fig. 2 shows the morphology of the SS410 powder particles. The chemical composition of the SS410 powder was listed in Table 1. In order to observe and analyze the microstructure evolution at the joint region, the substrate material used in the experiment was SS316 which had similar thermophysical properties but different microstruc-

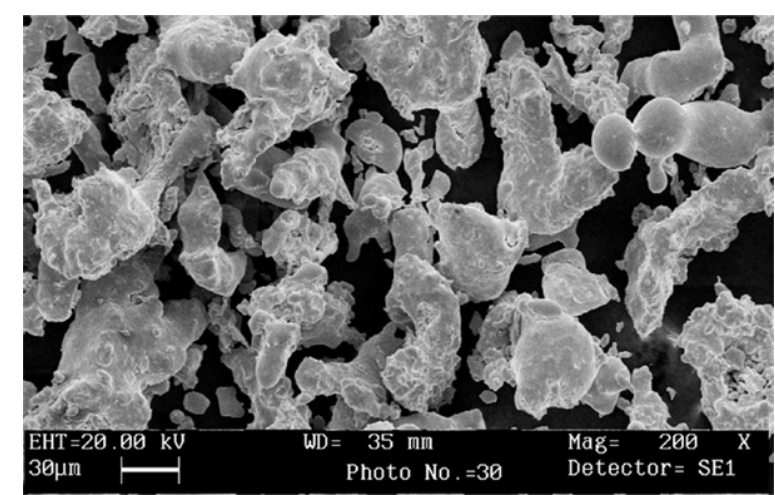

Fig. 2. Morphology of the SS410 powder particles. 


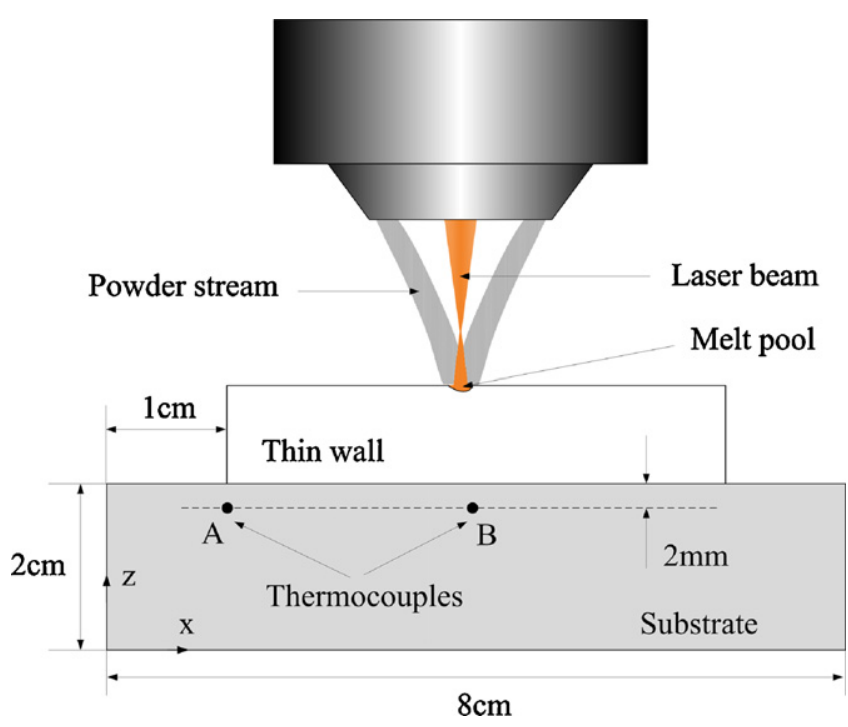

Fig. 3. Schematic diagram of the experiment.

ture evolution with SS410. The substrate plate's dimensions were $80 \times 40 \times 20 \mathrm{~mm}^{3}$.

The experiment of laser deposition SS410 thin wall with 200 layers was carried out with the laser materials processing system which mainly consisted of a $1000 \mathrm{~W}$ continuous-wave mode Nd: YAG laser, a five axes laser robot and a coaxial powder feed system. Two thermocouples were used to record the temperature during the process. To avoid the influence of shielding gas and powder particles outside the melt pool, the two thermocouples were inserted into the substrate to obtain the temperature readings at points $A$ and $B$. These two points were under the deposition path and the distance from the two points to the upper surface of substrate was $2 \mathrm{~mm}$, as shown in Fig. 3. The dimensions of the thin wall to be deposited were $60 \mathrm{~mm}$ (length) $\times 20 \mathrm{~mm}$ (height) $\times 3 \mathrm{~mm}$ (width).

When the deposition process began, the laser beam and nozzle travelled along the deposition path and SS410 powder was fed continuously into the melt pool. When one layer was deposited, the laser beam and nozzle were raised vertically upward by a Zincrement. Then the next layer was built on the previous one. When deposition process was finished, the part cooled down to room temperature.

Argon gas was used as powder carrier and shielding gas during the whole process. The gas flow rate was $10 \mathrm{~L} / \mathrm{min}$. Process parameters that result in less distortion and better geometry accuracy based on our previous work were used in this experiment. They were listed in Table 2.

The fabricated sample was cross-sectioned by wire-electrode cutting, polished using standard metallographic techniques and then etched with $\mathrm{HNO}_{3}: \mathrm{HF}: \mathrm{H}_{2} \mathrm{O}$ solution. Optical microscopy (OM) and scanning electron microscopy (SEM) coupled with an

Table 2

Process parameters used in the experiment.

\begin{tabular}{ll}
\hline Parameter & Value \\
\hline Laser power & $500 \mathrm{~W}$ \\
Laser travel speed & $2.5 \mathrm{~mm} / \mathrm{s}$ \\
Defocus distance & $9 \mathrm{~mm}$ \\
Beam radius & $1.5 \mathrm{~mm}$ \\
Z-increment between layers & $0.1 \mathrm{~mm}$ \\
Total number of layers & 200 \\
Powder feed rate & $0.72 \mathrm{~g} / \mathrm{min}$ \\
Shielding gas flow rate & $10 \mathrm{~L} / \mathrm{min}$ \\
\hline
\end{tabular}

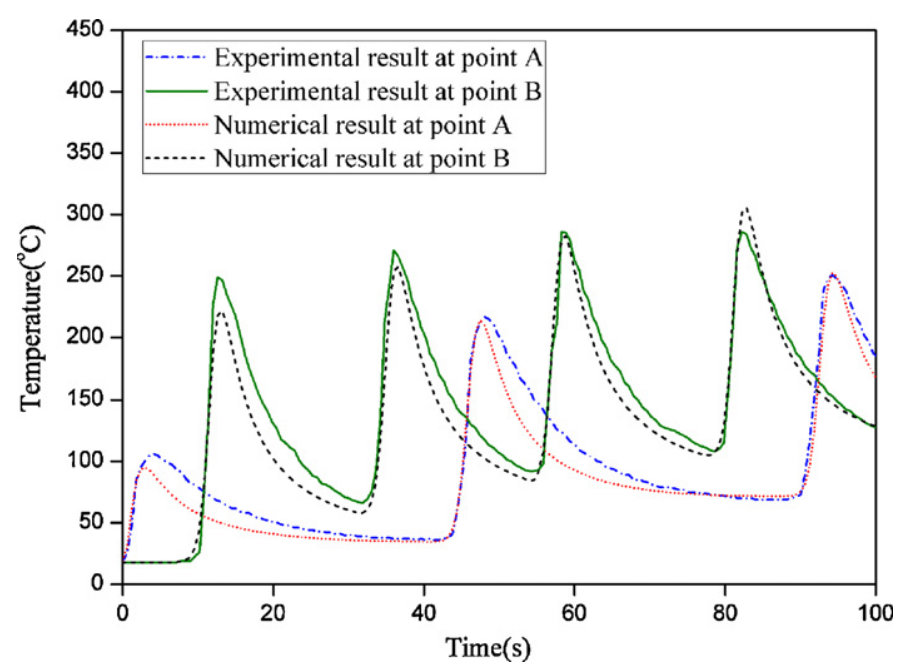

Fig. 4. Comparision between measured and simulated temperature history at points $\mathrm{A}$ and $\mathrm{B}$.

energy-dispersive spectrometer (EDS) were used to investigate the microstructure. X-ray diffraction (XRD) was used for phase identification. The hardness along the height centerline of the transverse (Y-Z) section was measured by a video display microhardness tester (HXD-1000B) using a 100 gf test force for a dwell time of $15 \mathrm{~s}$.

\section{Results and discussion}

\subsection{Model and experiment comparison}

Temperature readings at points $A$ and $B$ can be used to evaluate the 3D finite element model. In the model, the substrate material was set to SS316 and the thin wall material was set to SS410. The process parameters of the experiment were used in calculations. The calculated and measured temperature history at points A and $B$ were shown in Fig. 4. It can be seen that they have the same tendency. Nevertheless, the measured temperature decreased slower than the simulated one within the same thermal cycle. This may be caused by the assumption $\mathrm{A} 1$ that the thermal load in the model is a bit different from the actual laser energy distribution.

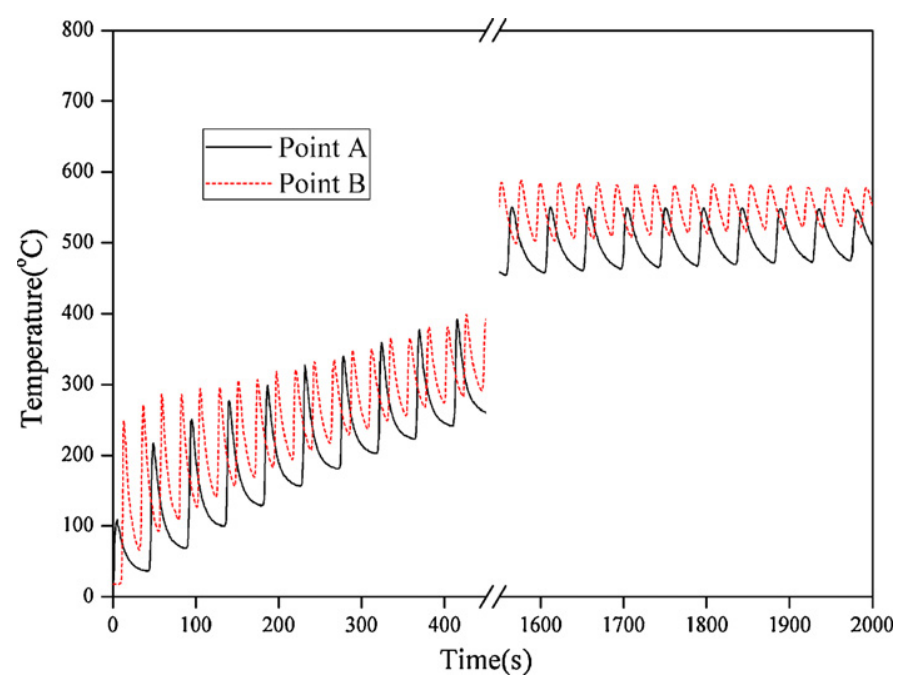

Fig. 5. Thermal history of points A and B in the substrate. 

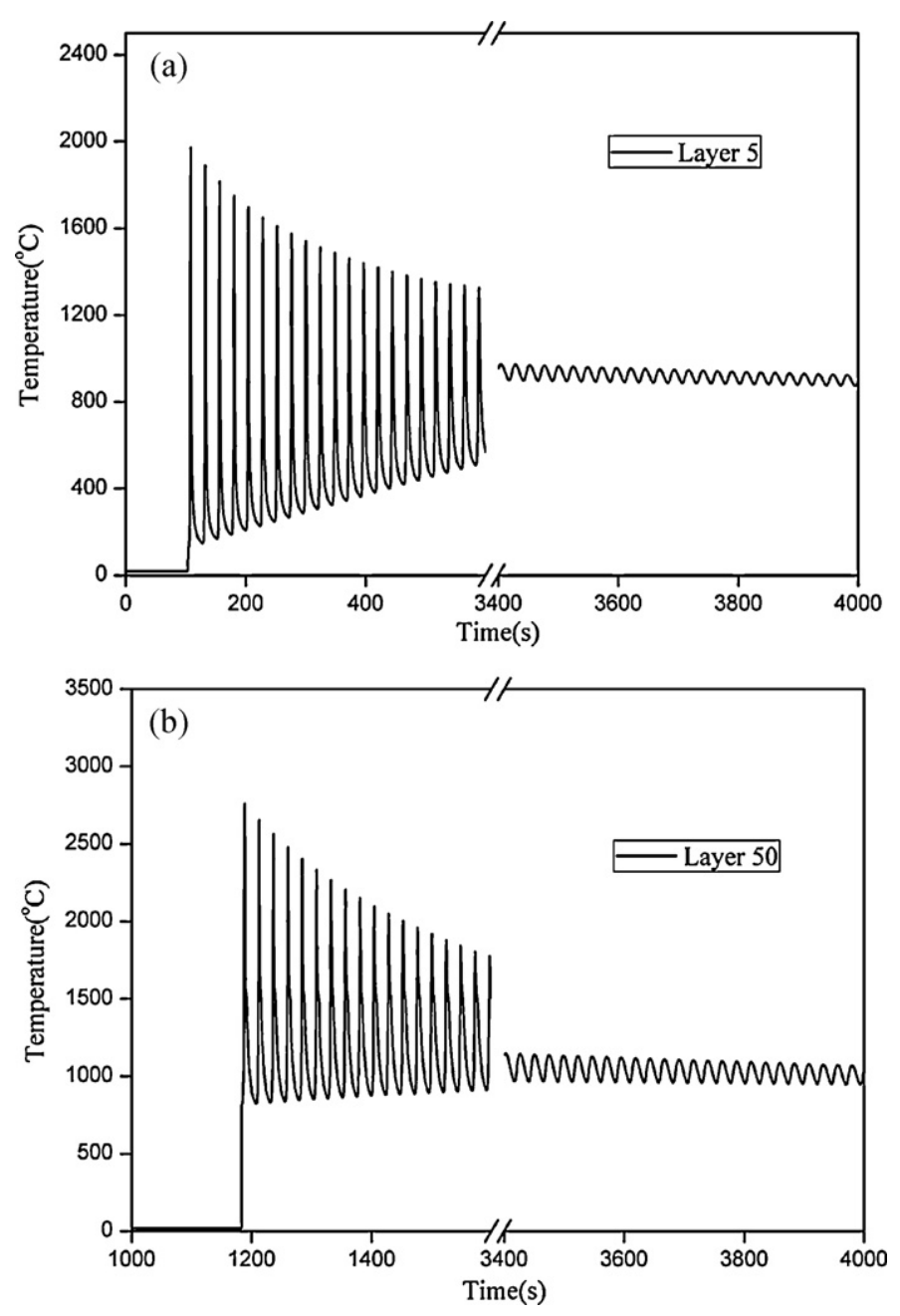

Fig. 6. Thermal history for the midpoints of (a) layer 5 and (b) layer 50.

\subsection{Thermal history}

Fig. 5 is the temperature history measured by the thermocouples. It can be seen that thermal history consists of a series of thermal cycles. The temperature reached a peak every time the laser beam passed over the measuring point, and then decreased to a resident temperature. Comparison of thermal history at points A and $B$ indicated that the thermal cycle peroid at point $A$ was the double of that at point B. However, thermal history at the two points had

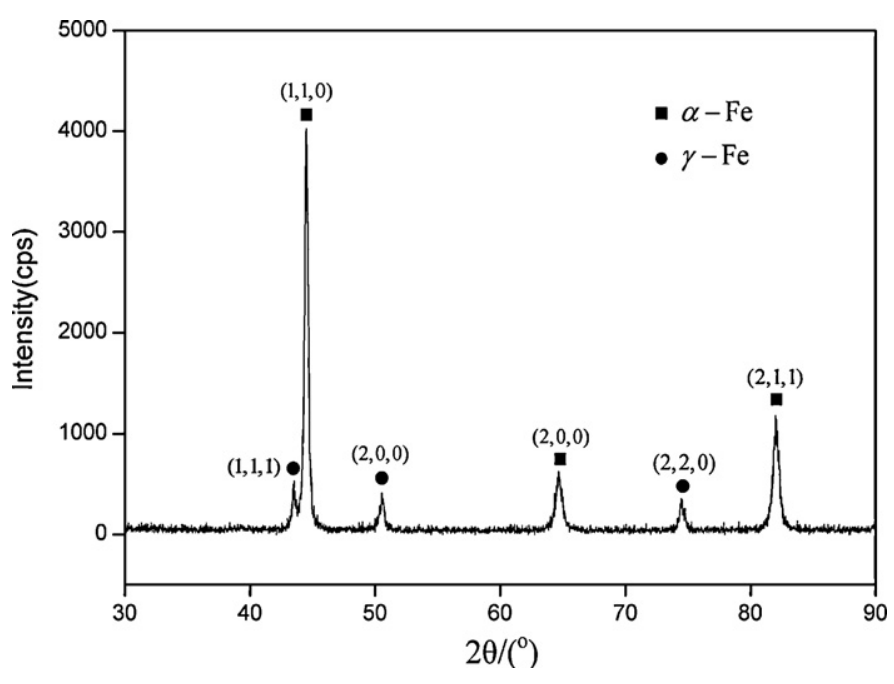

Fig. 8. The XRD pattern of Region I.

the same changing trend. During the initial stage, the peak and the resident temperature of the thermal cycle were low because of the heat dissipation by the cold substrate. They increased continually due to the integrated heat of laser irration. The fluctuations of temperaure tended to dampen as more layers were deposited. When the deposited material reached a certain height, the temperature fluctuated within a constant range. Wang et al. (2008) found the similar phenomena and they concluded that the decreasing laser power was required to keep a constant pool size as more layers were deposited.

The finite element model was used to calculate the temperature evolution during the deposition process. Fig. 6 shows the thermal history of material at midpoint of layer 5 and 50 . It was observed that fluctuations of the temperature tended to dampen as more SS410 powder atop this layer was deposited. Meanwhile, peak temperature and resident temperature tended to be closer. Finally, the temperature fluctuated within a small range. In the substrate and the thin tall, temperature history of the material was tending to be closer over time, which revealed that the absorption and loss of heat of the substrate and deposited material tended toward stability over time during the process.

\subsection{Microstructure examinations}

Microstrucure of cross section A-A, as shown in Fig. 7(a), was investigated to obtain the microstructure distribution along the
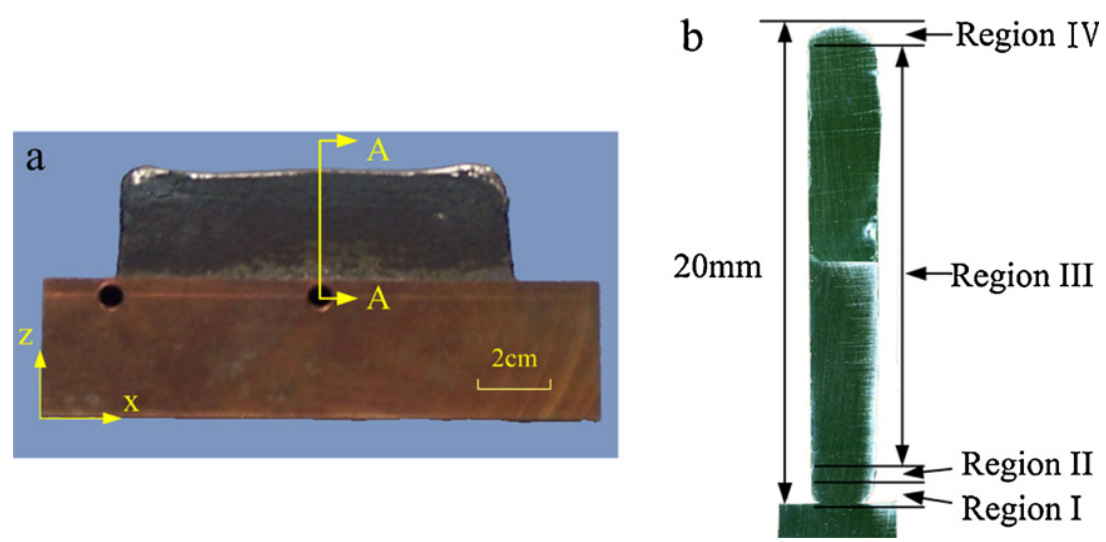

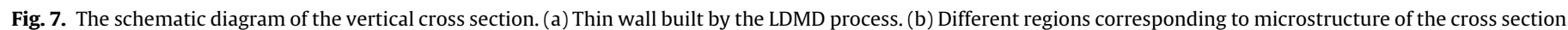
A-A. 


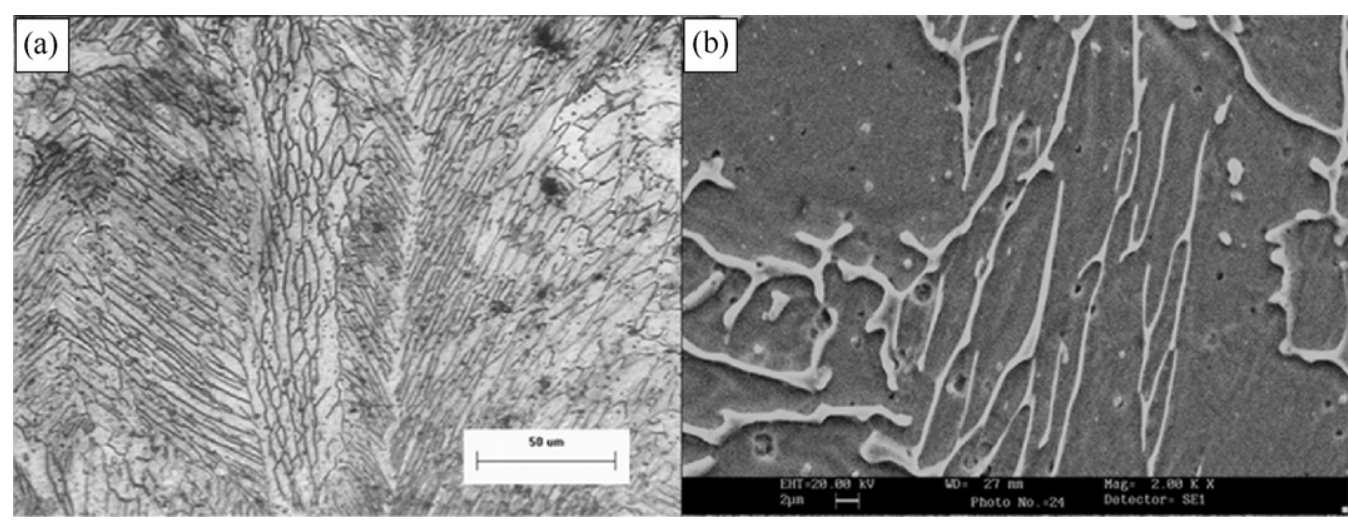

Fig. 9. Microstructure in Region I. (a) OM micrograph of the microstructure in Region I. (b) The corresponding SEM micrograph.

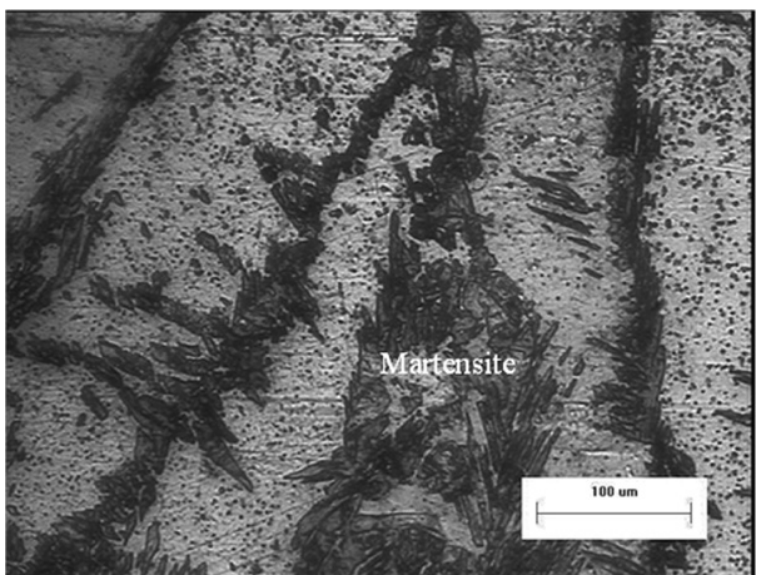

Fig. 10. OM micrograph of Region II.

height direction. The microstructure of cross section A-A was observed by OM and SEM. According to the microstrucure, cross section A-A can be seperated into four different regions, as shown in Fig. 7(b).

Region I is the joint region of the thin wall and the substrate. The height of this region is about $2 \mathrm{~mm}$. Fig. 8 is the XRD pattern of this region. The XRD result revealed that the phases in this region mainly consisted of ferrite $(\alpha-\mathrm{Fe})$ and retained austenite $(\gamma-\mathrm{Fe})$. Fig. 9 shows the OM and SEM images of typical microstructure in this region. It can be seen from Fig. 9(a) that the joint region consists of light matrix (background) and lamellar (dark lines) microstructure. The SEM image (Fig. 9(b)) revealed that the microstructure in this region was mixture of the ferrite matrix and retained austensite.

Region II is a transition region of about $0.5 \mathrm{~mm}$ height. Microstructure in this region is presented in Fig. 10. It can be seen that Region II consists of a mixture of ferrite matrix and martensite. The austenite was formed in the temperature range from Ac1 (the temperature at which austenite begins to form during heating) to austenization temperature $A c 3$. Due to the high cooling rate, austenite underwent a martensitic transforamtion during the cooling. It can be seen from Fig. 6(a) that after numerous thermal cycles, the temperature fluctuates within a small range and the cooling rate become small. As a result, there was a small quantity of retained martensite in Region II.

In Region III, the microstructure became uniform, as shown in Fig. 11. The microstructure completely consisted of refined ferrite matrix and some particles (Fig. 11(b)). EDS examination results of the matrix and particles are presented in Fig. 12. The element content of the ferrite matrix is: Fe-79.67, Cr-15.09, Si1.39, 0-3.29, Al-0.32, Mn-0.23 (wt\%). And the element content of the particles is Fe-43.97, Cr-11.18, Si-13.29, O-28.87, Al-1.47, Mn-1.22 (wt\%). It can be seen that the content of oxygen and silicon in the particles is higher than that in the ferrite matrix. Combined with the result of XRD, the main component of the particles is $\mathrm{SiO}_{2}$. Because the particles were very tiny, the EDS could not focus on the particles' surface entirely. The content of Fe element and $\mathrm{Cr}$ element in the EDS spectrum may be the result of the ferrite matrix. The high content of silicon can promote ferrite in martensitic stainless steels. It can be inferred that after repeated remelting, the material's temperature was higher than Ac3 temeprature before cooling down to room temperature slowly. The refined and uniform ferrite formed during the annealing process.

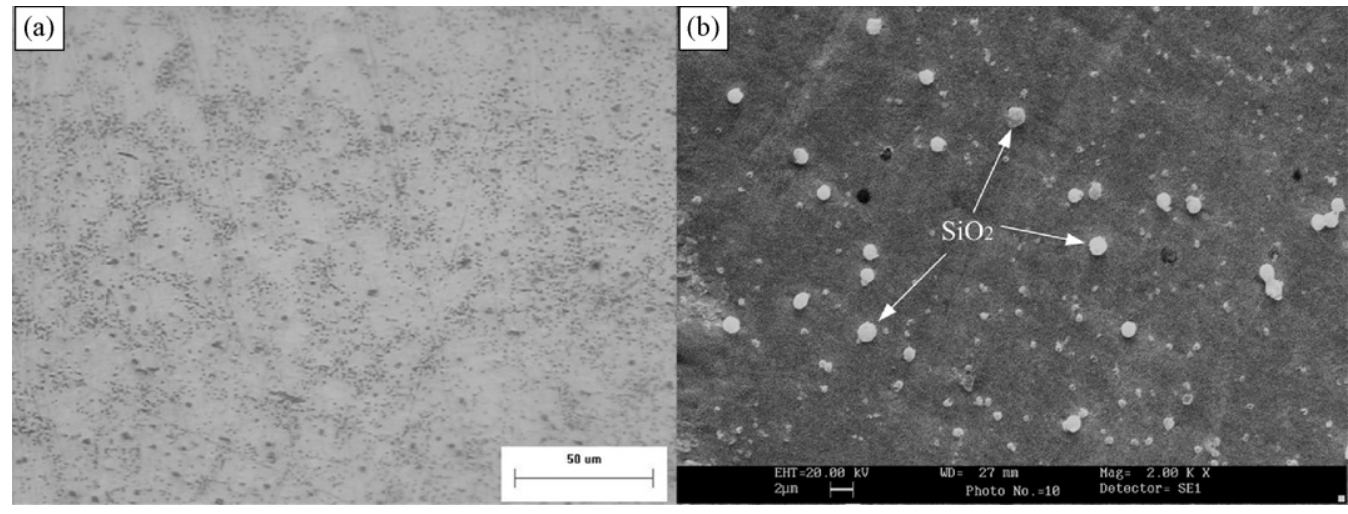

Fig. 11. Microstructure in Region III. (a) OM and (b) SEM micrograph. 

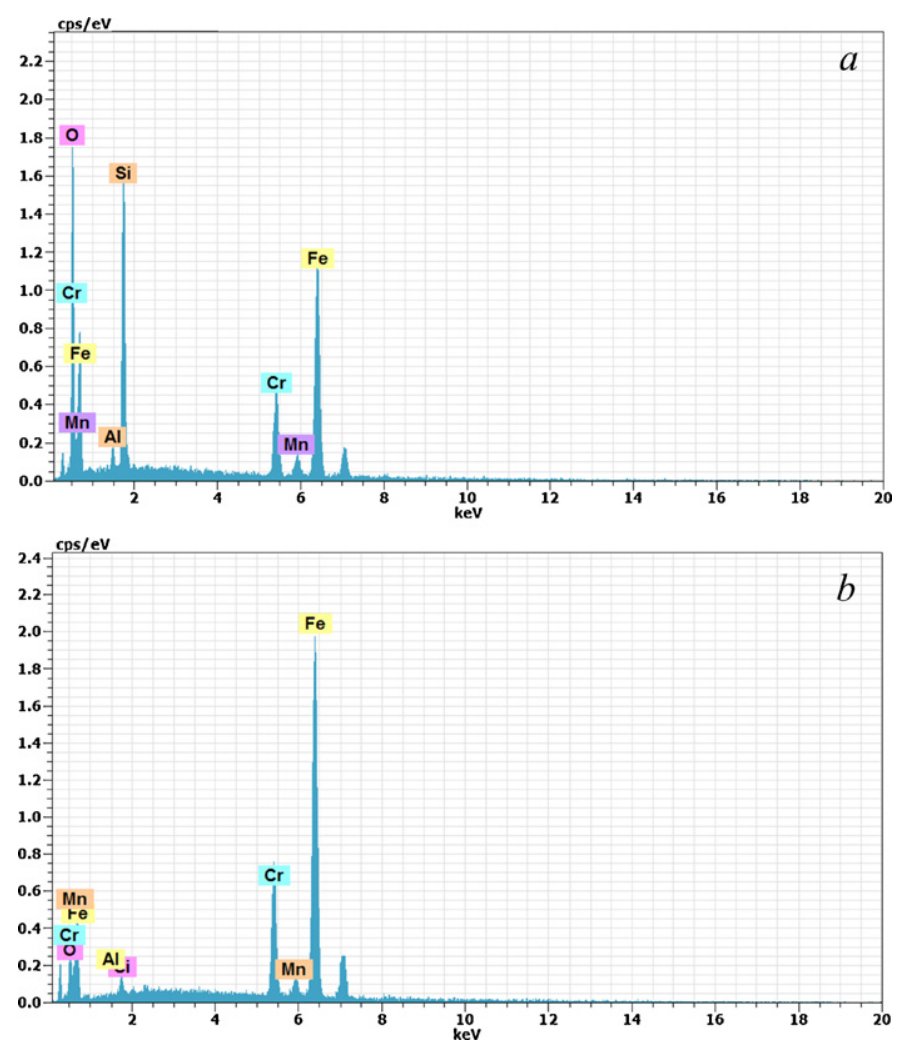

Fig. 12. EDS spectrum of the matrix (a) and particles (b) in Region III.

Region IV contained the last few layers of the thin wall. Material in this region experienced few thermal cycles and then cooled down to the room temperature. As shown in Fig. 13, the microstructure in this region consisted of columnar grain. When the last layer was deposited, liquid metal solidified and the dendrites grew along the heat flow direction due to the high temperature gradient in the melt pool. As the columnar dendritic grains grew, the cooling rate of the liquid metal decreased and the temperature tended to be uniform.

As described above, the distribution of microstructure in the thin wall is different from the assumption presented by Wang and Felicelli (2007). In that reference, the phase transformations that may occur in the material were predicted using semi-empirical models due to the high cooling rate in the laser deposition of ten layers SS410. This indicates that microstructure mainly depends on the thermal history material experienced. Before deposition, the temperature of substrate was at room temperature. When the

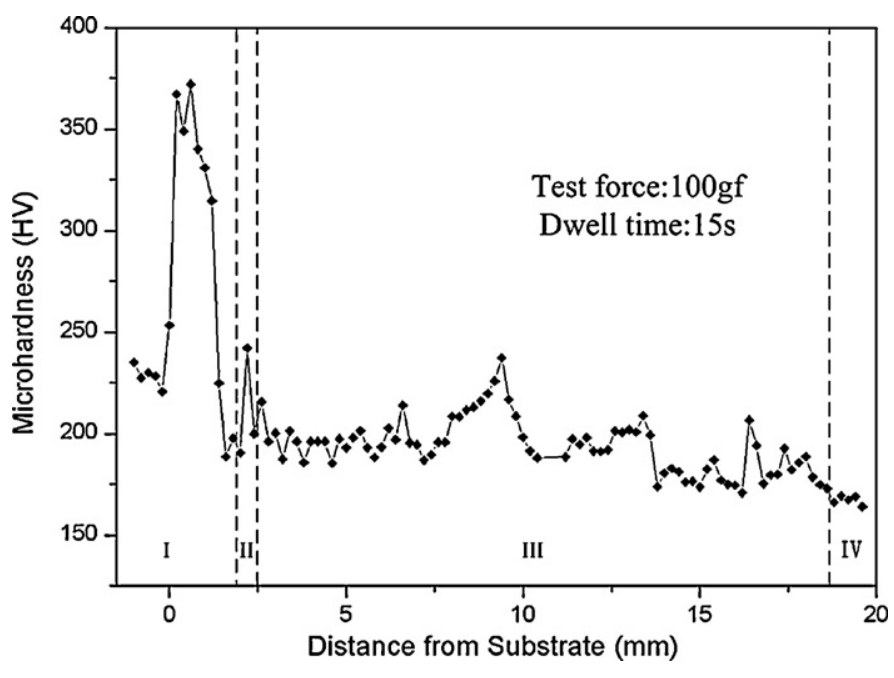

Fig. 14. Hardness distribution along the centerline of cross section A-A.

deposition process began, the average temperature of the substrate increased gradually with the laser irradiation. Solidification and remelting of SS410 powder resulted in the microstructure evolution from austenite, martensite to ferrite. When the absorption and loss of heat tended to be close to equilibrium, the microstructure in the thin wall became uniform and homogeneous.

\subsection{Microhardness}

Fig. 14 shows the hardness distribution along the centerline of cross section A-A. The results confirmed the microstructure evolution in the thin wall. It can be seen that the substrate hardness was about $230 \mathrm{HV}$, which was the same as the hardness before deposition. The maximum hardness value occurred in the joint region in which microstructure consisted of ferrite and retained austenite. It was higher than $350 \mathrm{HV}$. A sudden decrease of microhardness occurred at the $2 \mathrm{~mm}$ height of the thin wall. Because there was a small quantity of martensite in Region II with low height, Fig. 14 did not reflect the high hardness change in Region II. Then the microhardness in the region higher than $2.5 \mathrm{~mm}$ was homogeneous and about $200 \mathrm{HV}$. The phase in this region was mainly ferrite. It was confirmed that the microstructure had significant effects on the final hardness of the material. The microhardness measurement results proved that the evolution of temperature field in the part had important influence on the microstructure and mechanical properties of the thin wall.

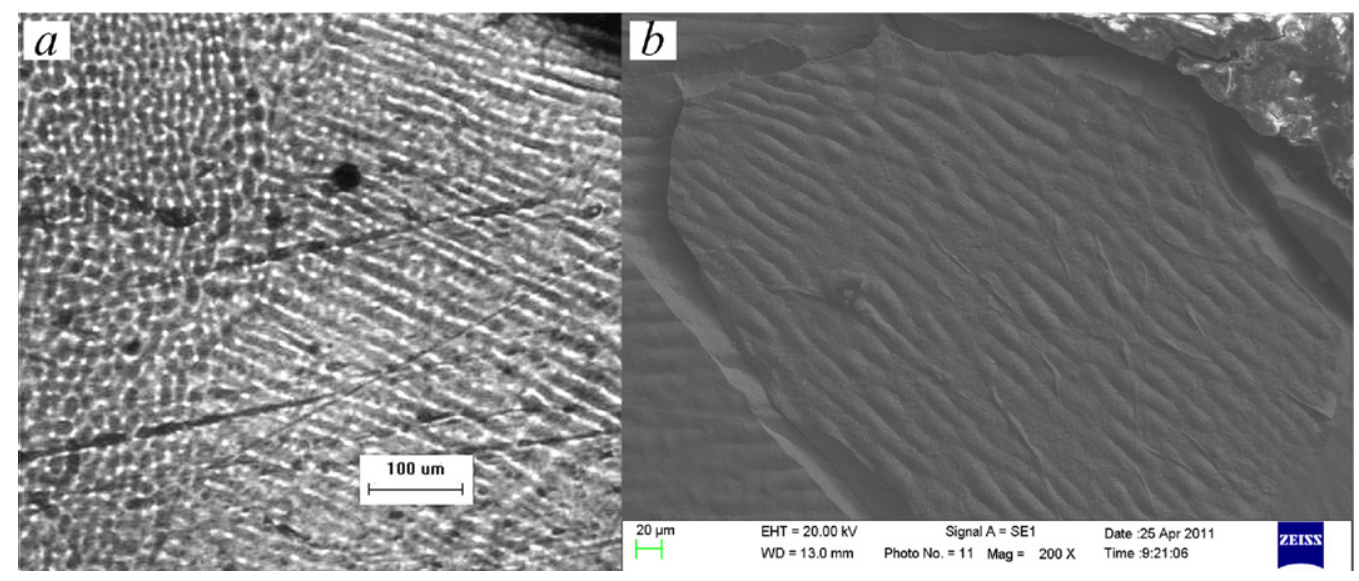

Fig. 13. OM (a) and SEM (b) micrograph of the top layers in the thin wall. 
For specific material, thermal history has important effects on the microstructure, and consequently on the final properties of the part. Meanwhile, the temperature evolution of the material depends significantly on the processing parameters. Thus, more uniform microstructure and better properties can be obtained by time-varying process parameters which result in the similar thermal history of all the deposited material. The time-varying process parameters can be determined by temperature prediction with numerical model, analyzing microstructure transformation and real-time temperature feedback. This is an efficient approach to improve the properties of the final part.

\section{Conclusions}

A comprehensive understanding of the thermal history, microstructure and hardness of the LDMD multilayer SS410 thin wall was obtained by experiments and numerical simulation. According to the simulated and experimental results, the main conclusions are summarized as follows:

During the LDMD process of multilayer SS410 thin wall, thermal history of the deposited material at different locations has the same changing trend. During the initial stage, the deposited material experiences a significant rapid quenching effect. Then the peak and resident temperature of thermal cycles tend to be close. When the deposited material reaches a certain height, the temperature fluctuates within a small range until the deposition is finished.
Thermal history of material has an important effect on the microstructure, and consequently on the final properties. In the multilayer SS410 thin wall built by LDMD process, the hardness of the joint region is higher than $350 \mathrm{HV}$. Microstructure turns to be homogeneous in the upper region of the thin wall and mainly consists of ferrite, the hardness of which is about $200 \mathrm{HV}$.

\section{Acknowledgements}

This work was supported by National Natural Science Foundation of China under Grant No. 10832011.

\section{References}

Alimardani, M., Toyserkani, E., Huissoon, J.P., 2007. A 3D dynamic numerical approach for temperature and thermal stress distributions in multilayer laser solid freeform fabrication. Opt. Lasers Eng. 45, 1115-1130.

Krishna, B.V., Bandyopadhyay, A., 2009. Surface modification of AISI 410 stainless steel using laser engineered net shaping. Mater. Des. 30, 1490-1496.

Pratt, P., Felicelli, S.D., Wang, L., Hubbard, C.R., 2008. Residual stress measurement of laser-engineered net shaping AISI 410 thin plates using neutron diffraction. Metall. Mater. Trans. A 39A, 3155-3163.

Wang, L., Felicelli, S., Gooroochurn, Y., Wang, P.T., Horstemeyer, M.F., 2008. Optimization of the LENS process for steady molten pool size. Mater. Sci. Eng. A 474, 148-156.

Wang, L., Felicelli, S., 2007. Process modeling in laser deposition of multilayer SS410 steel. J. Manuf. Sci. Eng. 129, 1028-1034.

Wang, L., Felicelli, S.D., Craig, J.E., 2009. Experimental and numerical study of the LENS rapid fabrication process. J. Manuf. Sci. Eng. 131, 1-8.

Yin, H., Wang, L., Felicelli, S.D., 2008. Comparison of two-dimensional and threedimensional thermal models of the LENS process. J. Heat Transfer 130, 1-7. 